\title{
Quality of life worsened the most severely in patients immediately after intensity-modulated radiotherapy for prostate cancer
}

This article was published in the following Dove Press journal: Research and Reports in Urology

\author{
Yasushi Nakai' \\ Nobumichi Tanaka' \\ Satoshi Anai' \\ Makito Miyake' \\ Isao Asakawa ${ }^{2}$ \\ Yosuke Morizawa' \\ Shunta Hori' \\ Kazumasa Torimoto' \\ Tomomi Fujii $^{3}$ \\ Masatoshi Hasegawa ${ }^{2}$ \\ Kiyohide Fujimoto'
}

'Department of Urology, Nara Medical University, Nara, Japan; ${ }^{2}$ Department of Radiation Oncology, Nara Medical University, Nara, Japan; ${ }^{3}$ Department of Pathology, Nara Medical University, Nara, Japan

Correspondence: Nobumichi Tanaka Department of Urology, Nara Medical University, 840 Shijo-cho, Nara 634-8522, Japan

Tel +8174422305 I ext 2338

Fax +81744229282

Email sendo@naramed-u.ac.jp
Purpose: The aim of this study was to evaluate the chronological changes in lower urinary tract symptoms (LUTSs), disease-related quality of life (QOL), and health-related QOL (HR-QOL) of patients who received intensity-modulated radiotherapy (IMRT).

Patients and methods: In 121 patients who had received IMRT and were followed up for $>2$ years, the International Prostate Symptom Score (IPSS), Overactive Bladder Symptom Score (OABSS), Expanded Prostate Cancer Index Composite (EPIC), and 8-Item Short-Form Health Survey (SF-8) were used before IMRT, at the halfway point in IMRT, immediately after IMRT, and 1-24 months after the completion of IMRT.

Results: The IPSS and OABSS and the urinary and bowel domains of the EPIC indicated that QOL worsened at the halfway point in IMRT, further worsened more severely immediately after IMRT, and then improved. The sexual domain of the EPIC significantly decreased at the halfway point in IMRT, which significantly lowered until 24 months. The scores of physical functioning, role physical, bodily pain, vitality, social functioning, and role emotional domains in the SF-8 significantly decreased and reached their lowest points immediately after IMRT.

Conclusion: QOL worsened the most severely in patients immediately after IMRT for prostate cancer. This knowledge can influence treatment recommendations and enable patients to make better informed decisions.

Keywords: chronological changes, intensity-modulated radiotherapy, prostate cancer, quality of life, radiotherapy

\section{Introduction}

The available modalities of treatment for prostate cancer (PCa) include open radical prostatectomy, robot-assisted radical prostatectomy (RARP), external beam radiotherapy (EBRT), high-dose radiation brachytherapy (BT), ${ }^{125} \mathrm{I}-\mathrm{BT}$, BT combined with EBRT, among others. Furthermore, focal therapy (high-intensity focused ultrasound, radiofrequency interstitial tumor ablation, and cryotherapy) has been performed for PCa. Each modality has its advantages and disadvantages and a significant impact on quality of life (QOL). ${ }^{1-5}$ Therefore, information on the QOL associated with each treatment is very important in treatment selection. Furthermore, knowledge of how QOL will change after each treatment is important for physicians and patients.

EBRT has been a standard treatment for PCa, especially for high-risk PCa, and is recommended in various guidelines, such as the European Association of Urology and National Comprehensive Cancer Network. Recently, intensity-modulated radiotherapy (IMRT) has replaced conventional EBRT and become a popular treatment for patients 
with clinically localized $\mathrm{PCa},{ }^{6-8}$ because IMRT has been shown to reduce toxicity with better biochemical control relative to those of conventional EBRT for $\mathrm{PCa} .{ }^{9}$

Lower urinary tract symptoms (LUTSs), disease-related QOL, and health-related QOL (HR-QOL) after IMRT have also been reported. ${ }^{10-13}$ However, these reports did not study QOL in the peri-treatment periods (during IMRT or immediately after IMRT). Previously, we reported time course changes in LUTSs, disease-related QOL, and HRQOL in patients who received salvage radiotherapy for biochemical recurrence of PCa and LUTSs and QOL worsen the most severely immediately after salvage radiotherapy. ${ }^{14}$ These results indicated that patients who received radiotherapy had worsened QOL in the peri-treatment period and it was very informative to know chronological changes of QOL including the peri-treatment periods (during IMRT and immediately after the completion of IMRT). Although Luo et $\mathrm{al}^{13}$ used Expanded PCa Index Composite (EPIC) to assess QOL associated with IMRT that included the period immediately after IMRT, and Berlin et al ${ }^{15}$ used EPIC to assess QOL associated with IMRT that included the period at 5 weeks after the start of IMRT, they did not use the other QOL assessment questionnaires or study on both the period during IMRT and the period immediately after the completion of IMRT. Consequently, information on QOL during these periods is not available.

In the present study, we assessed the chronological changes immediately before, during, and immediately after IMRT to treat LUTSs, HR-QOL, and disease-specific QOL during the 24 months following IMRT in patients treated for PCa by using the International Prostate Symptom Score (IPSS), overactive bladder symptom score (OABSS), EPIC, and Medical Outcomes Study 8-Item Short-Form Health Survey (SF-8) questionnaire.

\section{Patients and methods}

\section{Patients}

The study protocol was in accordance with the provisions of the Declaration of Helsinki (64th WMA General Assembly, Fortaleza, Brazil, in October 2013) and was approved by the ethics committee of Nara Medical University. Between April 2010 and March 2014, 141 consecutive patients underwent IMRT for localized PCa at the Nara Medical University Hospital. All patients in the study period were offered the opportunity to participate in this QOL study. The aims and methodologies of this study were explained to the patients, and then they were given the questionnaire and leaflet about this study. All patients who answered the questionnaire and sent back the questionnaire by post were considered as consented to participate in the present study and enrolled in this study. Initially, 141 patients were enrolled, but 20 were excluded because of the follow-up in other hospitals or insufficient data collection because of the cessation of follow-up visits during the 2 years after IMRT. The remaining 121 patients were analyzed. The clinicopathological characteristics of the enrolled patients are listed in Table 1.

\section{Treatments}

IMRT was given at a dose of 74-76 Gy in 2 Gy fractions using a micromultileaf collimator (Novalis; BrainLAB AG, Heimstetten, Germany). Image-guided radiotherapy using infrared-reflecting skin marker positioning and stereoscopic X-ray imaging was performed (ExacTrac rsp. Novalis Body; BrainLAB AG). In general, elective lymph node irradiation was not routinely employed during this study period, and the patients did not undergo pretreatment fiducial marker placement for image guidance during daily fractions. Neoadjuvant androgen deprivation therapy (ADT) was continued for 4 months and adjuvant ADT for 2 years; in addition, concomitant (8-week) ADT was continued during the radiation period.

Table I Characteristics of I 2 I patients who underwent IMRT

\begin{tabular}{ll}
\hline Variables & Total $(\mathbf{n = 1 2 I})$ \\
\hline Age at IMRT (median [range], years) & $73(55-82)$ \\
Initial PSA (median [range], ng/mL) & $13.1(3.1-2$ I8) \\
Gleason score at prostate biopsy & \\
6 & 14 \\
7 & 67 \\
8 & 13 \\
9 & 25 \\
I0 & 2 \\
NCCN risk classification & \\
Low & 12 \\
Intermediate & 52 \\
High & 57 \\
ADT & \\
Without ADT & 34 \\
Neoadjuvant therapy & 16 \\
Adjuvant therapy & 1 \\
Neoadjuvant and adjuvant therapy & 70 \\
Clinical T stage & \\
TI & 46 \\
T2 & 36 \\
T3 & 38 \\
T4 & 1 \\
Prescribed radiation dose (Gy) & 55 \\
76 & 66 \\
\hline Abbrevations: ADT, andogen deprvato thepy & \\
\hline
\end{tabular}

Abbreviations: $A D T$, androgen deprivation therapy; IMRT, intensity-modulated radiotherapy; NCCN, National Comprehensive Cancer Network; PSA, prostatespecific antigen. 


\section{$\mathrm{QOL}$}

The enrolled patients were assessed by IPSS ( $0-35$ scores, high score indicates disability), QOL question index (0-6 scores, high score indicates disability) and OABSS (0-15 scores, high score indicates disability) for LUTSs, EPIC (0-100 points, low score indicates disability) for disease-related QOL, and SF-8 (0-100 points, low score indicates disability) questionnaires for HR-QOL before IMRT initiation (baseline), 1 month after IMRT initiation (the halfway point in IMRT), immediately after the completion of IMRT (immediately after IMRT), and at $1,3,6,12$, and 24 months after the completion of IMRT. For the IPSS assessment, the sum of questions 1, 3, 5, and 6 and the sum of questions 2,4 , and 7 were defined as the voiding symptom-related sum and the storage symptom-related sum, respectively. ${ }^{15}$ Six years later from the beginning of this study, the collected data were evaluated.

\section{Statistical analyses}

Statistical analyses were performed by using SPSS for Windows (version 20.0; IBM Corporation, Armonk, NY, USA). To evaluate the change from baseline at each point in IPSS, QOL question index, OABSS, urinary, bowel, sexual, and hormone summary domain in EPIC, physical functioning, role physical, bodily pain, general health, vitality, social functioning, role emotional, and mental health in SF-8 questionnaire, Dunnett test was used considering multiple comparison. A $P$-value of $<0.05$ was considered as indicating statistical significance.

\section{Results}

\section{LUTSs following initiation of IMRT}

Total IPSS, QOL question index, and OABSS scores started to significantly increase at the halfway point in IMRT and then showed peaks immediately after the completion of IMRT. All the scores started to decrease at 1 month after the completion of IMRT, although the scores were still significantly higher than those at baseline (Figure 1).

Furthermore, the mean scores of the seven IPSS parameters, voiding symptom-related sum, and storage symptomrelated sum showed the highest immediately after the completion of IMRT (Table S1).

\section{Disease-related QOL following initiation of IMRT}

The score in the urinary domain summary started to significantly decrease at the halfway point in IMRT, then reached the
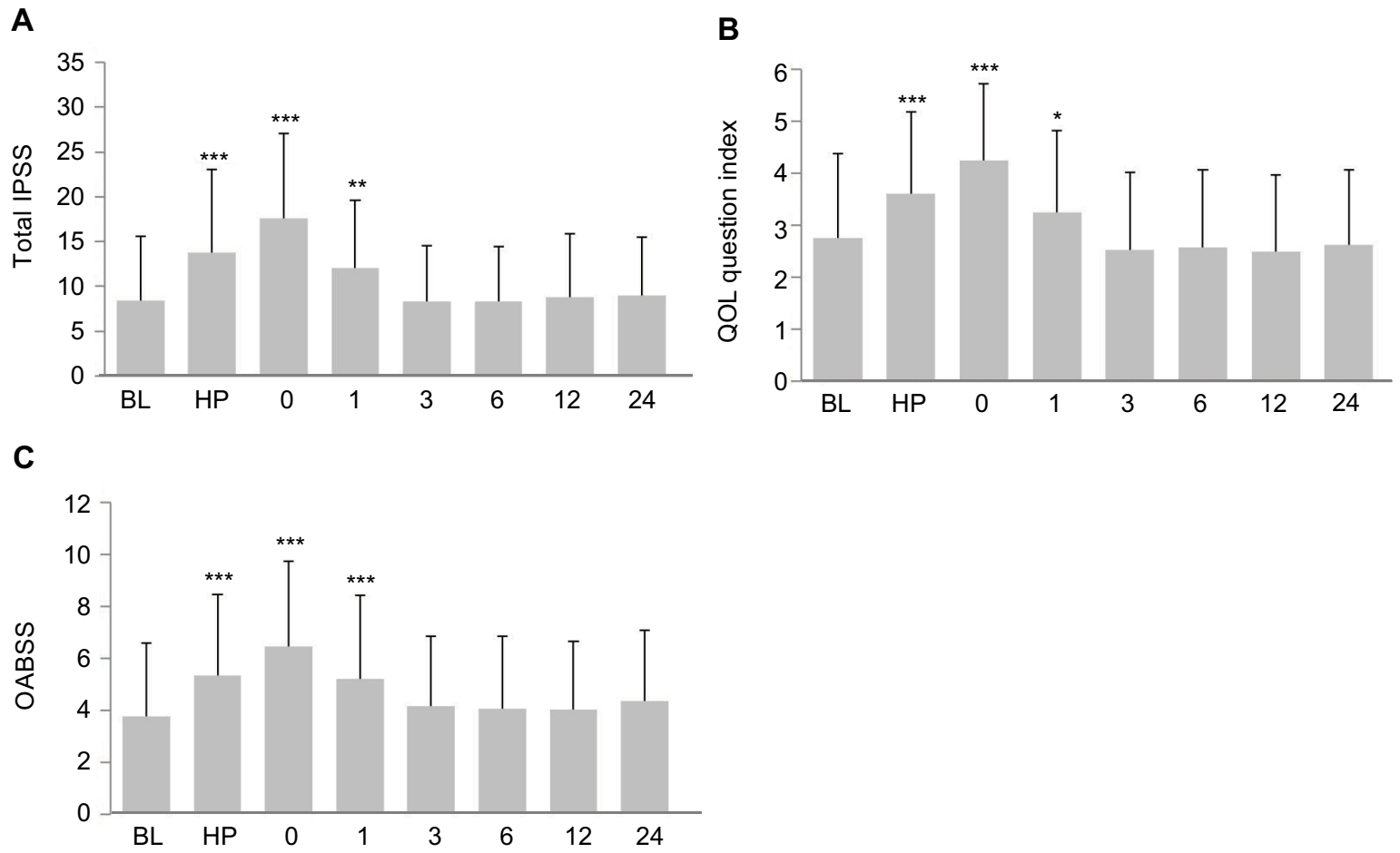

Figure I Chronological changes of IPSS (A), QOL question index (B), and OABSS (C).

Notes: $* P<0.05, * * P<0.01$, $* * * P<0.00$ I (compared to baseline using Dunnett test). HP, at the HP in IMRT; 0, immediately after IMRT; I, I month after IMRT; 3,3 months after IMRT; 6, 6 months after IMRT; 12, 12 months after IMRT; 24, 24 months after IMRT.

Abbreviations: BL, baseline; HP, halfway point; IMRT, intensity-modulated radiotherapy; IPSS, International Prostate Symptom Score; OABSS, Overactive Bladder Symptom Score; QOL, quality of life. 
lowest immediately after the completion of IMRT, and then started to improve by 1 month after the completion of IMRT, although these domain scores were still significantly lower than those at baseline (Figure 2A). Furthermore, the mean scores of urinary function, urinary bother, urinary irritative, and urinary incontinence showed the lowest immediately after the completion of IMRT (Table S2).

Bowel summary started to significantly decrease at the halfway point in IMRT, reached the lowest immediately after the completion of IMRT, and then started to improve at 1 month after the completion of IMRT, although these domain scores were still significantly lower than those at baseline (Figure 2). Furthermore, the mean scores of bowel function and bowel bother showed the lowest immediately after the completion of IMRT (Table S2).

The sexual summary significantly decreased at the halfway point in IMRT and was significantly lower until 24 months (Figure 2). The chronological changes of sexual summary domain in patients who did not undergo ADT $(n=34)$, underwent neoadjuvant therapy $(n=16)$, and underwent neoadjuvant plus adjuvant therapy $(n=70)$ are shown in Figure 3. The mean score of the sexual summary in patients who did not undergo ADT showed the lowest immediately after IMRT (Figure 3).

\section{A}

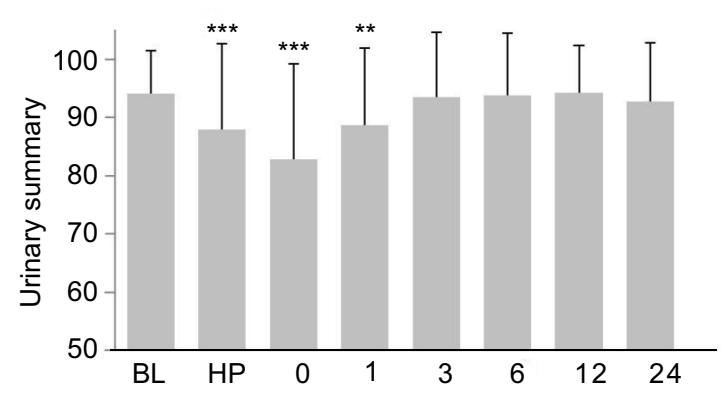

C

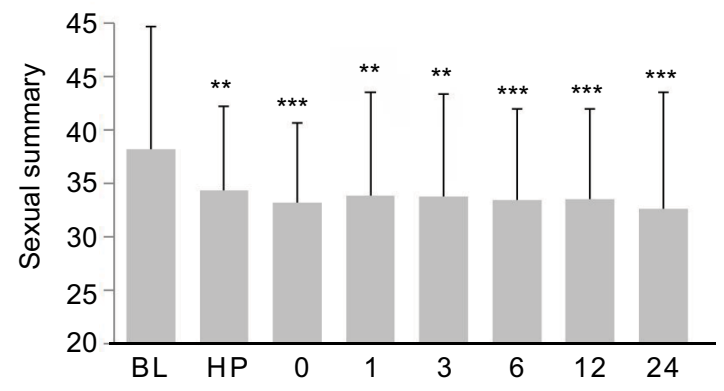

The hormone summary significantly decreased at 1 month and 24 months after the completion of IMRT (Figure 2). Figure 4 shows the chronological changes of hormone summary domain in patients who did not undergo ADT $(n=34)$, underwent neoadjuvant therapy $(n=16)$, and underwent neoadjuvant plus adjuvant therapy $(n=70)$. The mean scores of hormone summary score in patients who did not undergo ADT at the halfway point in IMRT, immediately after IMRT, and at 24 months after IMRT were $93.6( \pm 8.0), 93.9( \pm 7.3)$, and $92.0( \pm 9.3)$, respectively (Figure $4 \mathrm{~A})$.

\section{HRQOL following initiation of IMRT}

The scores of physical functioning, role physical, bodily pain, vitality, social functioning, and role emotional domains showed significant decrease and reached the lowest points immediately after IMRT. In the general health and mental health domain, the mean score immediately after IMRT showed the lowest although the scores immediately after IMRT did not decrease significantly compared to that at baseline (Figure 5; Table S3).

\section{Discussion}

The IPSS, QOL question index, and OABSS showed similar chronological changes. These scores started to increase

B

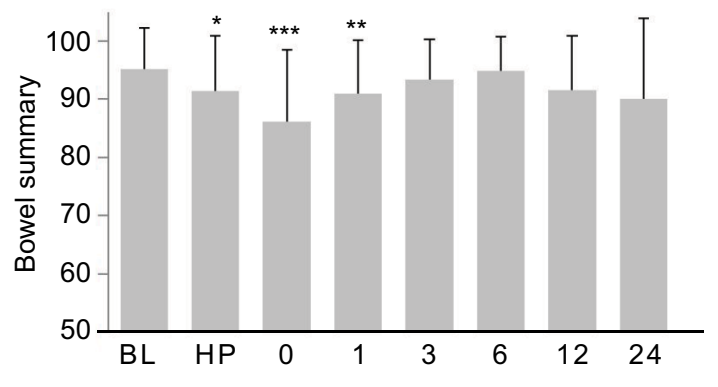

D

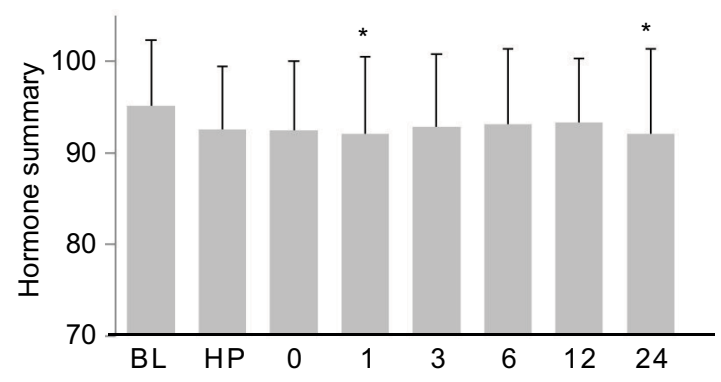

Figure 2 Chronological changes of urinary $(\mathbf{A})$, bowel (B), sexual (C), and hormone domain (D) summary score in EPIC.

Notes: $* P<0.05, * * P<0.0$ I, $* * * P<0.00$ I (compared to baseline using Dunnett test). HP, at the HP in IMRT; 0, immediately after IMRT; I, I month after IMRT; 3,3 months after IMRT; 6, 6 months after IMRT; I2, 12 months after IMRT; 24, 24 months after IMRT.

Abbreviations: BL, baseline; EPIC, Expanded Prostate Cancer Index Composite; HP, halfway point; IMRT, intensity-modulated radiotherapy. 
A

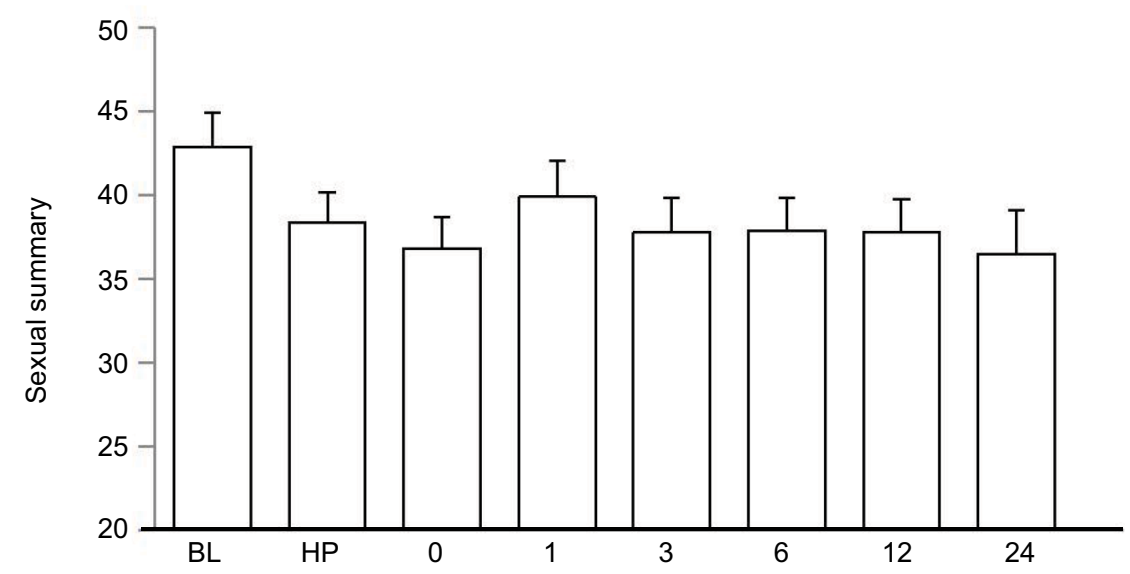

B

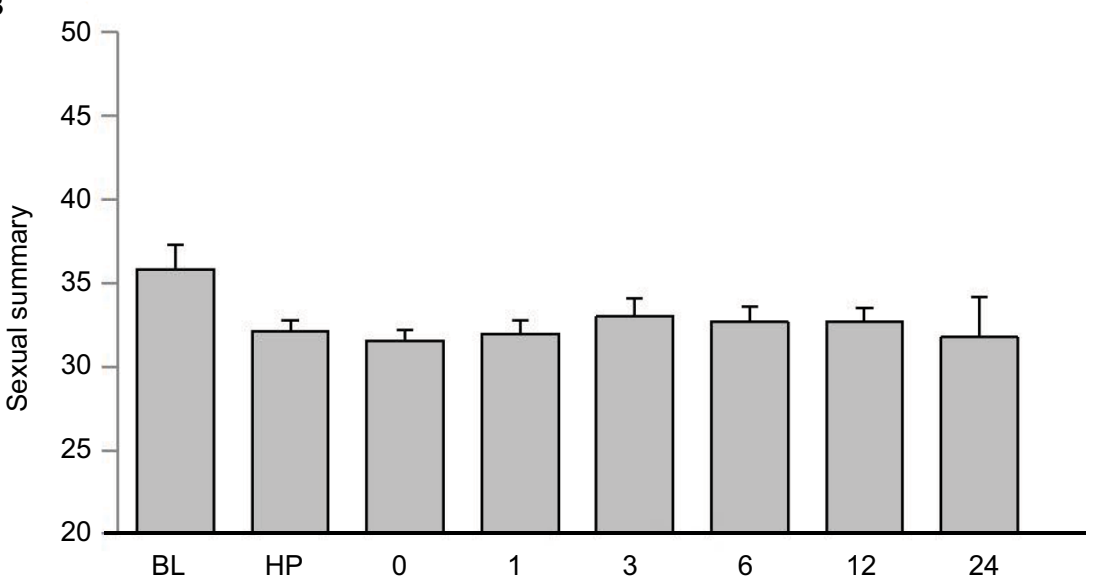

C

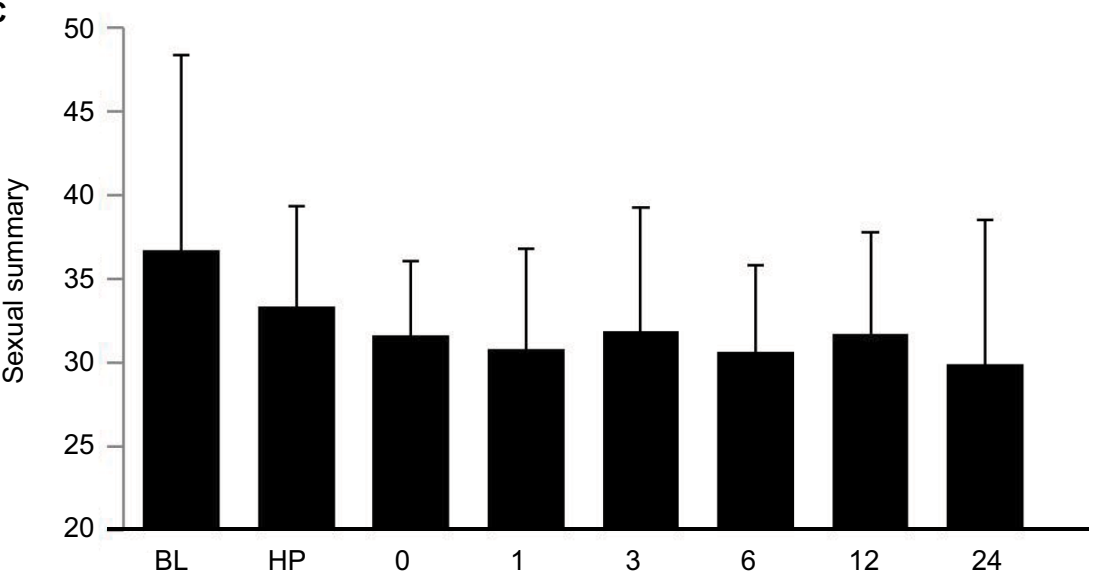

Figure 3 Chronological changes of the sexual summary score without ADT (A), with ADT as neoadjuvant therapy (B), and with ADT as neoadjuvant plus adjuvant therapy (C).

Notes: HP, at the HP in IMRT; 0, immediately after IMRT; I, I month after IMRT; 3, 3 months after IMRT; 6, 6 months after IMRT; I2, I2 months after IMRT; 24, 24 months after IMRT.

Abbreviations: ADT, androgen deprivation therapy; BL, baseline; HP, halfway point; IMRT, intensity-modulated radiotherapy. 
A

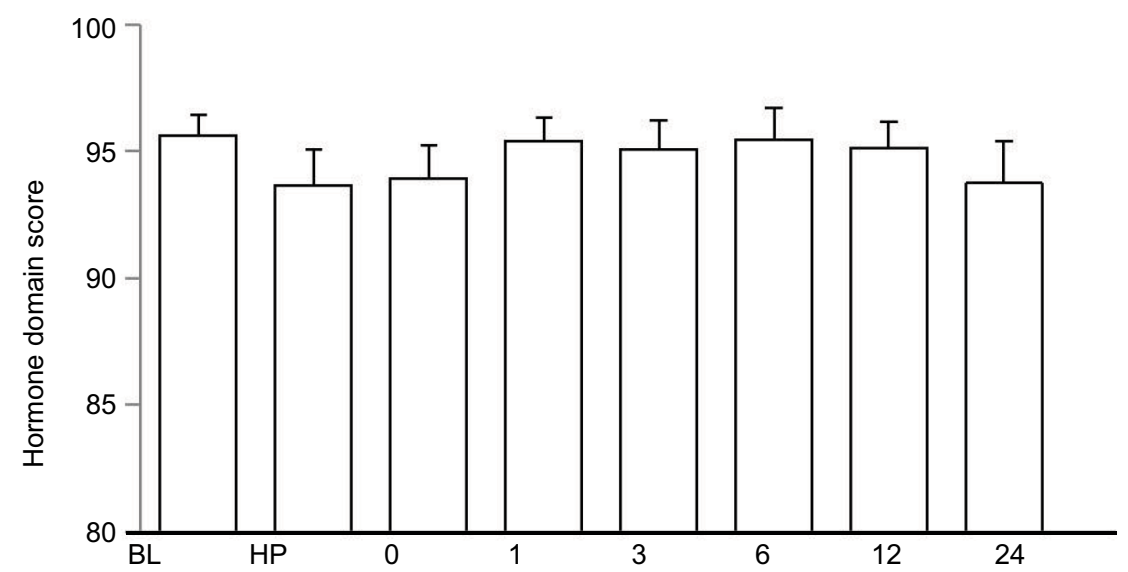

B

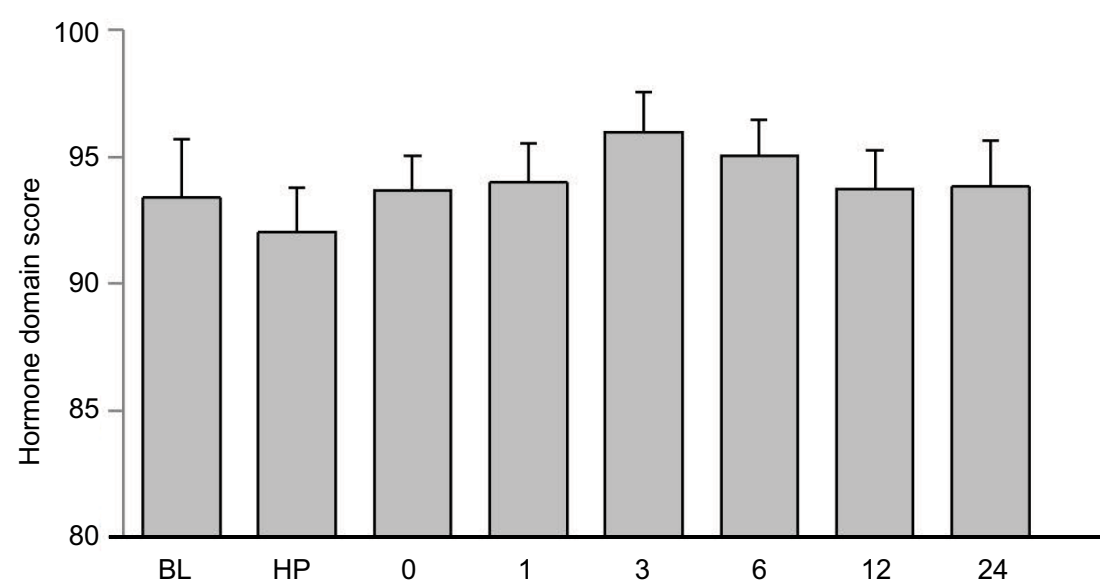

C

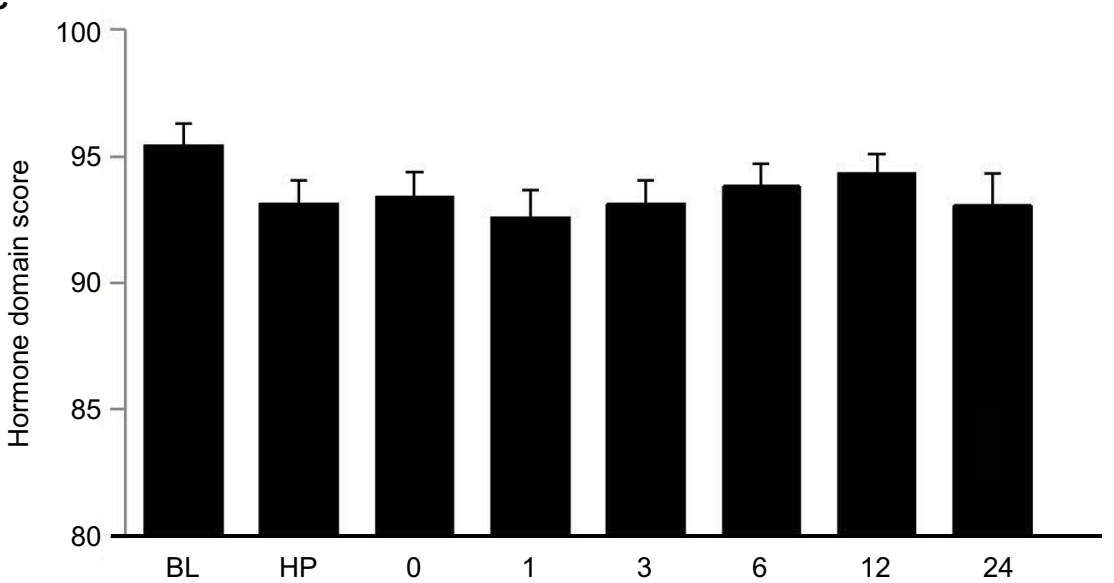

Figure 4 Chronological changes of the hormone summary score without ADT (A), with ADT as neoadjuvant therapy (B), and with ADT as neoadjuvant plus adjuvant therapy (C).

Notes: HP, at the HP in IMRT; 0, immediately after IMRT; I, I month after IMRT, 3, 3 months after IMRT; 6, 6 months after IMRT; I2, I2 months after IMRT; 24, 24 months after IMRT.

Abbreviations: ADT, androgen deprivation therapy; BL, baseline; HP, halfway point; IMRT, intensity-modulated radiotherapy. 
A

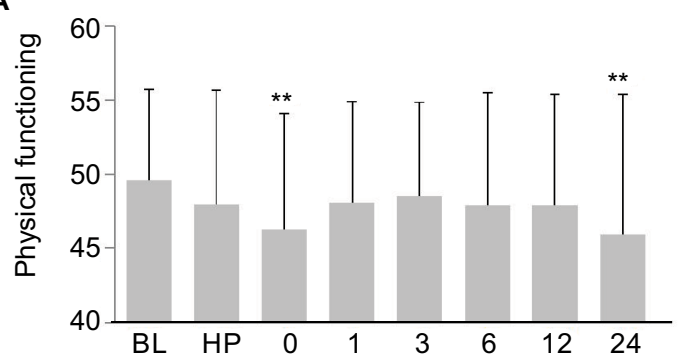

C

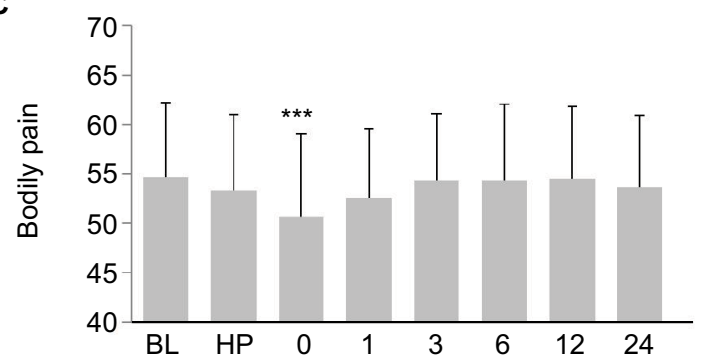

E
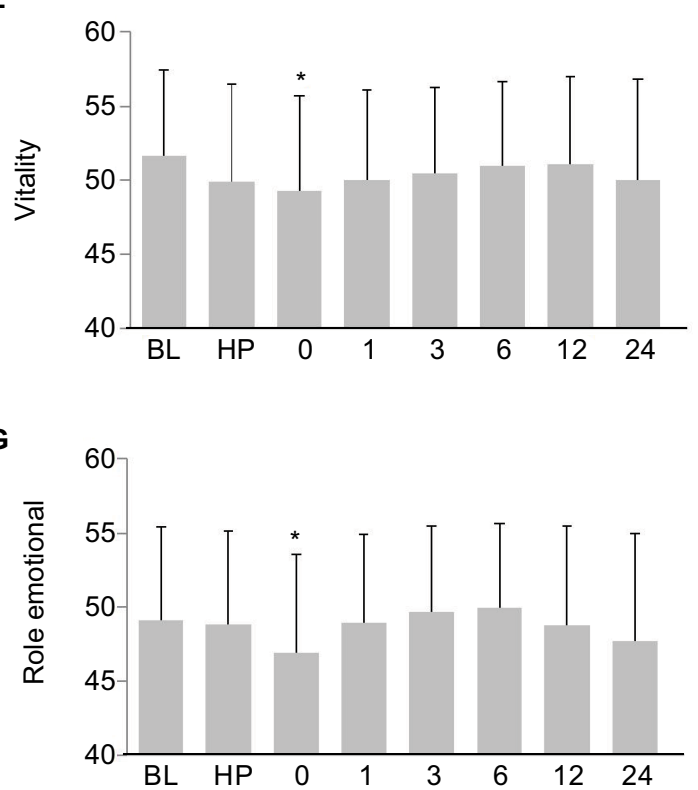

B
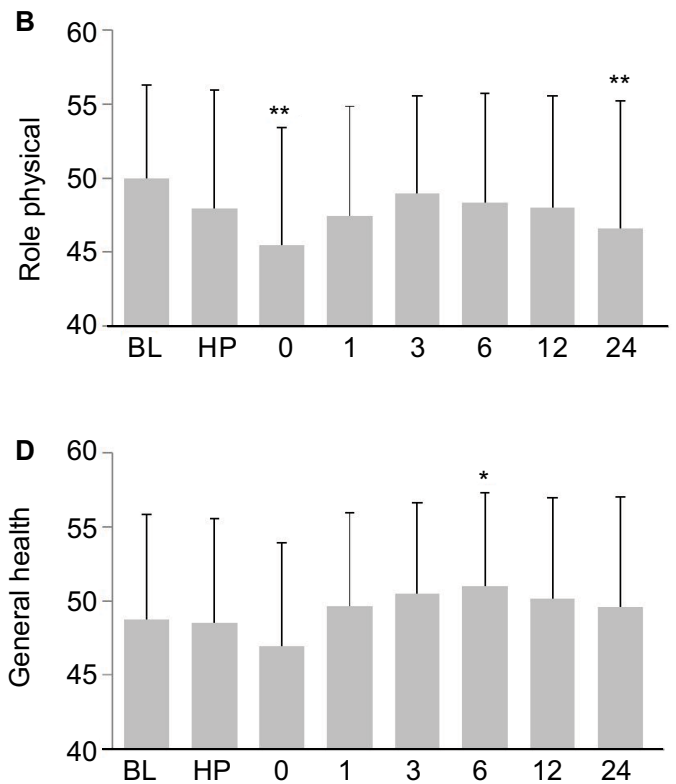

$\mathbf{F}$
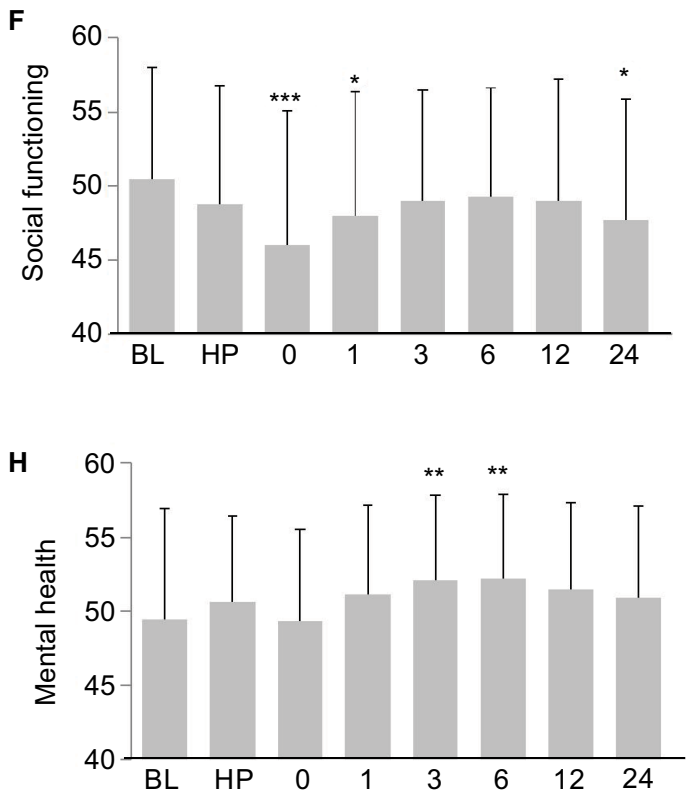

Figure 5 Chronological changes of physical functioning (A), role physical (B), bodily pain $(\mathbf{C})$, general health (D), vitality $(\mathbf{E})$, social functioning $(\mathbf{F})$, role emotional $(\mathbf{G})$, and mental health $(\mathbf{H})$ in SF-8.

Notes: $* P<0.05, * * P<0.0$ I, $* * * P<0.00$ I (compared to baseline using Dunnett test). HP, at the HP in IMRT; 0 , immediately after IMRT; I, I month after IMRT; 3,3 months after IMRT; 6, 6 months after IMRT; I2, 12 months after IMRT; 24, 24 months after IMRT.

Abbreviations: BL, baseline; HP, halfway point; IMRT, intensity-modulated radiotherapy.

during IMRT, showed peaks immediately after IMRT, and then started to decrease (improvement in QOL). To the best of our knowledge, there have been no reports about IPSS, QOL score, and OABSS assessments in the peri-treatment period (at the halfway point in IMRT and immediately after the completion of IMRT). ${ }^{16,17}$ Ghadjar et $\mathrm{al}^{17}$ reported that IPSS and QOL scores at 3, 6, 12, and 24 months after the completion of IMRT were almost the same at baseline. Chen et $\mathrm{al}^{18}$ reported that urinary symptoms worsened during treatment and were the worst at the completion of threedimensional conformal radiotherapy as measured by the $\mathrm{PCa}$ Symptoms Indices Questionnaire, although this was evaluated only during radiotherapy. These results are consistent with the results in the present study; that is, urinary symptoms were worst immediately after the completion of IMRT and then improved from 3 months after the completion of 
IMRT. Furthermore, the chronological changes in the urinary domain scores of the EPIC questionnaire were similar to the results of the IPSS, QOL score, and OABSS in the present study and indicated that urinary symptoms were worst immediately after the completion of IMRT. This chronological change in the urinary domain scores is similar to the IPSS, QOL score, and OABSS results in earlier reports and the EPIC results of other reports that included the periods during IMRT ${ }^{15}$ or immediately after IMRT. ${ }^{13}$ From these results, urination-related QOL initially worsened during IMRT, were worst immediately after IMRT, and then started to improve significantly from 6 months onward. Therefore, the severely worsened QOL can be overlooked if the QOL is estimated only after IMRT. It is important to estimate urination-related QOL in the peri-treatment period.

The EPIC bowel domain score started to worsen during IMRT, was worst immediately after IMRT, and then showed improvement at 3 months after the completion of IMRT in the present study. This chronological change was similar to that in the report of Luo et $\mathrm{al}^{13}$ that included the period immediately after IMRT. This result means the bowel-related QOL started to worsen during IMRT, was worst immediately after IMRT, and then started to improve. This result means that it is important to estimate bowel-related QOL in the peri-treatment period.

The sexual summary started to worsen during IMRT and was significantly lower until 24 months. However, ADT should be considered when the present results in sexual domain are evaluated. ${ }^{19}$ In the present study, $72 \%$ of patients received ADT, and it may be difficult to interpret the present data. Yamamoto et $\mathrm{al}^{12}$ reported that the sexual function and bother score gradually worsened at 3, 6, 12, and 24 months in patients who did not undergo ADT. King et $\mathrm{al}^{20}$ reported the change in sexual QOL in patients who underwent neoadjuvant ADT or did not undergo ADT. The patients who did not undergo ADT showed that the sexual QOL worsened with each passing year. On the other hand, the sexual QOL in patients who underwent ADT worsened at 1 month after stereotactic body radiotherapy and slightly improved, and then worsened with each passing year, although their report did not include results at the halfway point in IMRT or immediately after IMRT. Gaither et $\mathrm{al}^{21}$ reported that the prevalence of erectile dysfunction after radiotherapy (including brachytherapy and EBRT) was $34 \%$ at 1 year, $39 \%$ at 2 years, $44 \%$ at 3 years, and $57 \%$ at 5.5 years, and there was no significant difference between BT and EBRT using meta-analysis. In the present study, the patients who did not undergo ADT $(n=34)$ showed the lowest sexual QOL score immediately after IMRT (Figure 3 ). Considering the present study and other reports, the sexual domain-related QOL in patients who underwent IMRT without ADT worsened during IMRT, then recovered a little, then gradually worsened again. This change during IMRT may be caused by the decreased HR-QOL, time, and social restriction resulting from IMRT procedure; however, it is difficult to show the factors that caused this change from the data in the present study.

All domains except for mental health in the SF-8 questionnaire showed time course changes similar to those in the IPSS, OABSS, and EPIC urinary and bowel domains. That is, the QOL scores were worst immediately after IMRT for 24 months after the completion of IMRT. To the best of our knowledge, there is no report on HR-QOL after IMRT that includes the periods during IMRT and immediately after IMRT. ${ }^{7,10-12,16}$ Those reports showed that HR-QOL worsened during the 1-3 months after IMRT. However, our results showed that HRQOL started to worsen during IMRT and was worst immediately after IMRT, but then recovered gradually.

In the present study, LUTSs, disease-specific QOL, and the HR-QOL worsened the most severely immediately after IMRT. This means that treatments to improve QOL during IMRT may be needed, particularly for LUTSs, bowel function, and sexual function. However, few studies of treatments for LUTSs, bowel function, and sexual function after IMRT have been reported. Palumbo et $\mathrm{al}^{22}$ reported the efficacy of calcium channel blockers and statins for acute rectal toxicity during IMRT. Zelefsky et $\mathrm{al}^{23}$ proved the efficacy of sildenafil citrate for sexual function after radiotherapy (EBRT and BT). These agents may be considered during IMRT. We have found no reports on treatments for LUTSs after IMRT but have found some reports on treatments for LUTSs after BT. ${ }^{24,25}$ Therefore, the efficacy of alpha-1 blockers or anticholinergic drugs for LUTSs during IMRT should be evaluated in the future.

Few well-constructed studies have assessed LUTSs, HRQOL, and disease-specific QOL following IMRT. This is the first study to evaluate the outcomes of IMRT at specific time points during IMRT and early in the posttreatment period in PCa patients. However, this observational study has several limitations, such as the cohort including patients with various stages of PCa, the small number of patients, and short followup period. QOL after treatment can differ by stage in patients with PCa. ${ }^{26}$ More patients and longer follow-up period can be needed. Furthermore, the comparison to another treatment for PCa is lacked in the present study. Especially, comparison to RARP should be noted. The rate of stress incontinence 
after RARP was about $10-20 \%$ at 6 months. ${ }^{27}$ Furthermore, effective sling for stress incontinence has been reported. ${ }^{28}$ In this situation, difference in QOL between radiotherapy and RARP should be evaluated in the future. There are some limitations in the present study. However, we believe that this study provides important insights concerning chronological changes in LUTSs, disease-specific QOL, and HR-QOL for patients receiving IMRT. A better understanding of the QOL outcomes associated with IMRT may enable patients to make better informed decisions regarding treatment for PCa.

\section{Conclusion}

Quality of life worsened the most severely in patients immediately after intensity-modulated radiotherapy for prostate cancer. This knowledge can influence treatment recommendations and enable patients to make better-informed decisions.

\section{Acknowledgment}

The authors would like to thank R Okamura (Department of Surgery, Kyoto University Hospital) for assistance with statistical analysis.

\section{Author contributions}

YN interpreted the data and drafted the manuscript. NT conceived the study, participated in the study design, and revised the manuscript. IA collected the data and performed the radiotherapy treatments. SA collected the data. MM collected the data and edited the manuscript. SH and YM collected the data. TF performed pathologic diagnosis. $\mathrm{MH}$ provided valuable help and performed the radiotherapy treatments. KF participated in the study design. All authors contributed to data analysis, drafting and revising the article, gave final approval of the version to be published, and agree to be accountable for all aspects of the work.

\section{Disclosure}

The authors report no conflicts of interest in this work.

\section{References}

1. Sanda MG, Dunn RL, Michalski J, et al. Quality of life and satisfaction with outcome among prostate-cancer survivors. $N$ Engl J. Med. 2008;358(12):1250-1261.

2. Chen RC, Clark JA, Talcott JA. Individualizing quality-of-life outcomes reporting: how localized prostate cancer treatments affect patients with different levels of baseline urinary, bowel, and sexual function. J Clin Oncol. 2009;27(24):3916-3922.

3. Yaxley JW, Coughlin GD, Chambers SK, et al. Robot-assisted laparoscopic prostatectomy versus open radical retropubic prostatectomy early outcomes from a randomised controlled phase 3 study. Lancet. 2016;388(10049):1057-1066.
4. Werneburg GT, Kongnyuy M, Halpern DM, et al. Effects of focal vs total cryotherapy and minimum tumor temperature on patient-reported quality of life compared with active surveillance in patients with prostate cancer. Urology. 2018;113:110-118.

5. Valerio M, Cerantola Y, Eggener SE, et al. New and established technology in focal ablation of the prostate: a systematic review. Eur Urol. 2017;71(1):17-34.

6. Shinohara N, Maruyama S, Shimizu S, et al. Longitudinal comparison of quality of life after real-time tumor-tracking intensity-modulated radiotherapy and radical prostatectomy in patients with localized prostate cancer. J Radiat Res. 2013;54(6):1095-1101.

7. Goineau A, Marchand V, Rigaud J, et al. Prospective evaluation of quality of life 54 months after high-dose intensity-modulated radiotherapy for localized prostate cancer. Radiat Oncol. 2013;8:53.

8. Tanaka N,Asakawa I, NakaiY, et al. Comparison of PSA value at last followup of patients who underwent low-dose rate brachytherapy and intensitymodulated radiotherapy for prostate cancer. BMC Cancer. 2017;17(1):573.

9. Yu T, Zhang Q, Zheng T, et al. The effectiveness of intensity modulated radiotherapy versus three-dimensional radiotherapy in prostate cancer: a meta-analysis of the literatures. PLoS One. 2016;11(5):e0154499.

10. Namiki S, Ishidoya S, Ito A, et al. Five-year follow-up of health-related quality of life after intensity-modulated radiotherapy for prostate cancer. Jpn J Clin Oncol. 2009;39(11):732-738.

11. Lips I, Dehnad H, Kruger AB, et al. Health-related quality of life in patients with locally advanced prostate cancer after 76 Gy intensitymodulated radiotherapy vs. 70 Gy conformal radiotherapy in a prospective and longitudinal study. Int J Radiat Oncol Biol Phys. 2007;69(3):656-661.

12. Yamamoto S, Fujii Y, Masuda H, et al. Longitudinal change in health-related quality of life after intensity-modulated radiation monotherapy for clinically localized prostate cancer. Qual Life Res. 2014;23(5):1641-1650.

13. Luo HC, Cheng LP, Cheng HH, et al. Long-term quality of life outcomes in patients with locally advanced prostate cancer after intensity-modulated radiotherapy combined with androgen deprivation. Med Oncol. 2014;31(6):991.

14. Miyake M, Tanaka N, Asakawa I, et al. Changes in lower urinary tract symptoms and quality of life after salvage radiotherapy for biochemical recurrence of prostate cancer. Radiother Oncol. 2015;115(3):321-326.

15. Berlin A, Cho E, Kong V, et al. Phase 2 trial of guideline-based postoperative image guided intensity modulated radiotherapy for prostate cancer: Toxicity, biochemical, and patient-reported health-related quality-of-life outcomes. Pract Radiat Oncol. 2015;5(5):e473-e482.

16. Corbin KS, Kunnavakkam R, Eggener SE, Liauw SL. Intensity modulated radiotherapy after radical prostatectomy: Early results show no decline in urinary continence, gastrointestinal, or sexual quality of life. Pract Radiat Oncol. 2013;3(2):138-44.

17. Ghadjar P, Jackson A, Spratt DE, et al. Patterns and predictors of amelioration of genitourinary toxicity after high-dose intensity-modulated radiotherapy for localized prostate cancer: implications for defining postradiotherapy urinary toxicity. Eur Urol. 2013;64(6):931-938.

18. Chen RC, Zhang Y, Chen MH, et al. Patient-reported quality of life during radiation treatment for localized prostate cancer: results from a prospective phase II trial. BJU Int. 2012;110(11):1690-1695.

19. Benedict C, Traeger L, Dahn JR, et al. Sexual bother in men with advanced prostate cancer undergoing androgen deprivation therapy. $J$ Sex Med. 2014;11(10):2571-2580.

20. King CR, Collins S, Fuller D, et al. Health-related quality of life after stereotactic body radiotherapy for localized prostate cancer: results from a multi-institutional consortium of prospective trials. Int J Radiat Oncol Biol Phys. 2013;87(5):939-945.

21. Gaither TW, Awad MA, Osterberg EC, et al. The Natural History of Erectile Dysfunction After Prostatic Radiotherapy: A Systematic Review and Meta-Analysis. J Sex Med. 2017;14(9):1071-1078.

22. Palumbo I, Matrone F, Montesi G, et al. Statins Protect Against Acute RT-related Rectal Toxicity in Patients with Prostate Cancer: An Observational Prospective Study. Anticancer Res. 2017;37(3):1453-1458. 
23. Zelefsky MJ, Shasha D, Branco RD, et al. Prophylactic sildenafil citrate improves select aspects of sexual function in men treated with radiotherapy for prostate cancer. J Urol. 2014;192(3):868-874.

24. Oyama N, AokiY, Ito H, et al. Alpha 1-adrenoceptor blocker may improve not only voiding but also storage lower urinary tract symptoms caused by (125) I brachytherapy for prostate cancer. ISRN Urol. 2014;2014:1-8.

25. Tanaka N, Torimoto K, Asakawa I, et al. Use of alpha-1 adrenoceptor antagonists in patients who underwent low-dose-rate brachytherapy for prostate cancer - a randomized controlled trial of silodosin versus naftopidil. Radiat Oncol. 2014;9:302.
26. Vanagas G, Mickeviciene A, Ulys A. Does quality of life of prostate cancer patients differ by stage and treatment? ScandJPublic Health. 2013;41(1):58-64.

27. Ficarra V, Novara G, Rosen RC, et al. Systematic review and metaanalysis of studies reporting urinary continence recovery after robotassisted radical prostatectomy. Eur Urol. 2012;62(3):405-417.

28. Ferro M, Bottero D, D'Elia C, et al. Virtue male sling for post-prostatectomy stress incontinence: a prospective evaluation and mid-term outcomes. BJU Int. 2017;119(3):482-488. 


\section{Supplementary materials}

Table SI Time course changes in IPSS, QOL question score, and OABSS after IMRT

\begin{tabular}{|c|c|c|c|c|c|c|c|c|}
\hline \multirow[t]{2}{*}{ Questionnaire } & \multirow[t]{2}{*}{ Baseline } & \multirow{2}{*}{$\frac{\text { During }}{\text { IMRT }}$} & \multirow{2}{*}{$\frac{\text { Immediately }}{\text { After IMRT }}$} & \multicolumn{5}{|c|}{ Follow-up (months) } \\
\hline & & & & $\mathbf{I}$ & 3 & 6 & 12 & 24 \\
\hline \multicolumn{9}{|l|}{ IPSS questions } \\
\hline I. Emptying & $\mathrm{I} .0(\mathrm{I} .3)$ & $1.7(1.8)$ & $2.1(1.8)$ & $1.2(1.5)$ & $0.8(1.2)$ & $0.9(1.4)$ & $0.9(1.4)$ & I.I (I.5) \\
\hline 2. Frequency & $1.6(1.5)$ & $2.3(1.7)$ & $3.1(1.7)$ & $2.2(1.6)$ & $1.6(1.5)$ & $1.6(1.5)$ & $1.7(1.6)$ & $1.7(1.7)$ \\
\hline 3. Intermittency & $1.0(1.5)$ & $1.7(1.8)$ & $2.3(1.9)$ & $1.6(1.7)$ & $1.0(1.5)$ & $0.9(1.5)$ & I.I (I.5) & $1.2(1.6)$ \\
\hline 4. Urgency & $0.9(1.4)$ & $\mathrm{I} .4(\mathrm{I} .7)$ & $2.1(1.9)$ & $1.6(1.7)$ & $0.9(1.3)$ & $\mathrm{I} .0(1.3)$ & $1.0(1.3)$ & $1.0(1.4)$ \\
\hline 5. Weak stream & $\mathrm{I} .5(\mathrm{I} .7)$ & $2.5(2.0)$ & $3.1(1.9)$ & $2.1(1.9)$ & $\mathrm{I} .4(\mathrm{I} .6)$ & $1.5(\mathrm{I} .6)$ & $1.6(1.8)$ & $1.7(1.8)$ \\
\hline 6. Hesitancy & $0.9(1.5)$ & $1.6(1.9)$ & $2.0(2.0)$ & I.2(I.7) & $0.9(1.4)$ & $0.8(1.4)$ & I.0 (I.5) & $1.0(1.4)$ \\
\hline 7. Nocturia & $2.0(1.3)$ & $2.8(1.4)$ & $3.1(1.3)$ & $2.6(1.3)$ & $2.2(1.3)$ & $2.2(1.2)$ & $2.2(1.2)$ & $2.3(1.3)$ \\
\hline IPSS I+3+5+6 (voiding symptoms) & $4.3(5.1)$ & $7.6(6.2)$ & $9.4(6.3)$ & $6.2(5.4)$ & $4.2(4.6)$ & $4.0(4.5)$ & $4.6(5.1)$ & $4.9(4.9)$ \\
\hline IPSS 2+4+7 (storage symptoms) & $4.4(3.4)$ & $6.6(3.8)$ & $8.0(4.3)$ & $6.3(3.8)$ & $4.9(3.1)$ & $4.6(3.2)$ & $4.8(3.3)$ & $4.7(3.4)$ \\
\hline Total IPSS & $8.9(7.8)$ & $14.2(9.2)$ & $17.8(9.4)$ & $12.6(8.3)$ & $9.2(7.0)$ & $8.8(7.0)$ & $9.6(7.7)$ & $9.9(7.6)$ \\
\hline QOL score & $2.7(1.7)$ & $3.6(1.6)$ & $4.2(1.5)$ & $3.3(1.6)$ & $2.6(1.5)$ & $2.6(1.5)$ & $2.6(1.5)$ & $2.6(1.5)$ \\
\hline OABSS & $3.8(2.8)$ & $5.3(3.0)$ & $6.6(3.3)$ & $5.4(3.3)$ & $4.4(2.8)$ & $4.3(3.0)$ & $4.2(2.8)$ & $4.3(2.8)$ \\
\hline
\end{tabular}

Note: SD in parentheses.

Abbreviations: IMRT, intensity-modulated radiotherapy; IPSS, International Prostate Symptom Score; OABSS, Overactive Bladder Symptom Score; QOL, quality of life.

Table S2 Time course changes in EPIC questionnaire score after IMRT

\begin{tabular}{|c|c|c|c|c|c|c|c|c|}
\hline \multirow[t]{2}{*}{ Domains } & \multirow[t]{2}{*}{ Baseline } & \multirow{2}{*}{$\frac{\text { During }}{\text { IMRT }}$} & \multirow{2}{*}{$\frac{\text { Immediately }}{\text { After IMRT }}$} & \multicolumn{5}{|c|}{ Follow-up (months) } \\
\hline & & & & I & 3 & 6 & 12 & 24 \\
\hline Urinary summary & $94.1(7.4)$ & $89.4(I I .3)$ & $83.9(14.0)$ & $90.0(10.5)$ & $94.2(7.4)$ & $94.5(6.5)$ & $94.2(8.1)$ & $92.8(10.0)$ \\
\hline I. Urinary function & $94.1(10.2)$ & 91.1 (12.7) & $84.7(16.6)$ & $89.2(13.3)$ & $94.6(9.7)$ & $94.4(9.3)$ & $94.5(10.3)$ & $92.2(13.8)$ \\
\hline 2. Urinary bother & $94.2(8.2)$ & $88.3(13.1)$ & $83.3(14.6)$ & $89.8(10.8)$ & $93.9(7.6)$ & $94.6(6.8)$ & $94.0(8.0)$ & $93.2(8.8)$ \\
\hline 3. Urinary irritative & $95.9(7.6)$ & $91.0(12.3)$ & $85.1(14.9)$ & $92.0(9.7)$ & $96.3(5.8)$ & $96.6(6.1)$ & $95.8(7.7)$ & $95.8(7.3)$ \\
\hline 4. Urinary incontinence & $94.4(11.0)$ & $92.1(13.8)$ & $88.2(16.8)$ & $90.0(15.8)$ & $94.1(12.2)$ & $94.4(10.9)$ & $94.6(12.0)$ & $90.1(16.1)$ \\
\hline Bowel summary & $95.2(7.2)$ & $91.8(9.2)$ & $86.1(12.5)$ & $90.1(9.3)$ & $93.4(6.8)$ & $95.0(5.8)$ & $91.6(9.3)$ & 90.4 (13.0) \\
\hline I. Bowel function & $92.8(9.8)$ & $87.9(12.5)$ & $81.3(15.4)$ & $87.1(12.1)$ & $90.0(9.8)$ & $92.4(8.8)$ & $87.9(12.3)$ & $87.7(14.6)$ \\
\hline 2. Bowel bother & $97.5(7.4)$ & $95.7(7.4)$ & $90.8(I I .4)$ & $94.8(8.0)$ & $96.8(5.0)$ & $97.5(3.8)$ & $95.3(7.8)$ & 93.1 (12.7) \\
\hline Sexual summary & $38.2(11.6)$ & $34.4(8.1)$ & $33.0(7.1)$ & $33.9(9.8)$ & $33.7(9.6)$ & $33.5(8.7)$ & $33.5(8.4)$ & $32.5(10.3)$ \\
\hline I. Sexual function & $12.6(17.1)$ & $7.5(12.8)$ & $5.8(11.1)$ & $7.2(13.8)$ & $6.9(14.0)$ & $6.4(13.5)$ & $6.9(12.8)$ & $7.0(13.1)$ \\
\hline 2. Sexual bother & $95.8(11.0)$ & $94.9(12.3)$ & $94.1(14.6)$ & $93.9(16.0)$ & $94.1(15.9)$ & $94.6(15.2)$ & $93.5(15.2)$ & $90.1(21.7)$ \\
\hline Hormone summary & $95.1(7.1)$ & $92.6(6.9)$ & $92.4(7.6)$ & $92.0(8.4)$ & $92.8(7.9)$ & $93.1(8.2)$ & $93.3(7.0)$ & $92.0(9.3)$ \\
\hline I. Hormone function & $90.3(10.5)$ & $86.0(12.5)$ & $86.3(13.3)$ & $86.5(12.7)$ & $87.0(14.0)$ & $87.7(13.6)$ & $88.5(11.5)$ & $87.0(13.2)$ \\
\hline 2. Hormone bother & $99.0(2.6)$ & $98.1(3.9)$ & $97.6(4.5)$ & $97.4(6.3)$ & $98.0(4.7)$ & $97.7(5.7)$ & $97.3(6.2)$ & $96.6(7.5)$ \\
\hline
\end{tabular}

Note: SD in parentheses.

Abbreviations: EPIC, Expanded Prostate Cancer Index Composite; IMRT, intensity-modulated radiotherapy.

Table S3 Time course changes in SF-8 after IMRT

\begin{tabular}{|c|c|c|c|c|c|c|c|c|}
\hline \multirow[t]{2}{*}{ Domains } & \multirow[t]{2}{*}{ Baseline } & \multirow{2}{*}{$\frac{\text { During }}{\text { IMRT }}$} & \multirow{2}{*}{$\frac{\text { Immediately }}{\text { After IMRT }}$} & \multicolumn{5}{|c|}{ Follow-up (months) } \\
\hline & & & & $\mathbf{I}$ & 3 & 6 & 12 & 24 \\
\hline Physical functioning & $49.6(6.1)$ & $47.8(7.9)$ & $46.3(8.0)$ & $48.0(6.9)$ & $48.5(6.4)$ & $48.0(7.5)$ & $48.1(7.3)$ & $46.0(9.1)$ \\
\hline Role physical & $50.0(6.3)$ & $48.0(8.2)$ & $45.5(8.0)$ & $47.5(7.4)$ & $48.9(6.6)$ & $48.5(7.2)$ & $48.1(7.4)$ & $46.6(8.4)$ \\
\hline Bodily pain & $54.2(7.6)$ & $53.5(7.5)$ & $50.9(8.2)$ & $52.6(7.0)$ & $54.4(6.8)$ & $54.3(7.8)$ & $54.6(7.3)$ & $53.6(7.6)$ \\
\hline General health & $48.8(6.8)$ & $48.7(7.0)$ & $47.1(7.0)$ & $49.8(6.1)$ & $50.4(6.2)$ & $51.0(6.3)$ & $50.2(6.7)$ & $50.0(7.1)$ \\
\hline Vitality & $51.8(5.6)$ & $50.0(6.6)$ & $49.4(6.4)$ & $50.2(6.0)$ & $50.6(5.7)$ & $51.0(5.6)$ & $51.3(5.8)$ & $50.1(6.5)$ \\
\hline Social functioning & $50.4(7.6)$ & $48.9(8.1)$ & $45.9(9.1)$ & $48.3(8.1)$ & $48.9(7.5)$ & $49.2(7.4)$ & $49.1(8.1)$ & $48.1(8.1)$ \\
\hline Role emotional & $49.0(6.4)$ & $48.7(6.4)$ & $46.8(6.7)$ & $49.0(6.0)$ & $49.7(5.6)$ & $50.0(5.7)$ & $48.8(6.6)$ & $48.4(6.9)$ \\
\hline Mental health & $49.5(7.5)$ & $50.1(5.8)$ & $49.4(6.1)$ & $51.2(6.0)$ & $52.1(5.8)$ & $52.2(5.7)$ & $51.5(5.8)$ & $50.1(6.2)$ \\
\hline PCS & $50.3(6.1)$ & $48.1(7.5)$ & $45.9(7.7)$ & $47.8(6.7)$ & $48.8(6.0)$ & $48.4(7.3)$ & $48.7(6.4)$ & $47.1(7.5)$ \\
\hline MCS & $48.4(7.3)$ & $49.1(6.1)$ & $47.8(6.2)$ & $49.5(6.4)$ & $50.0(5.7)$ & $50.5(5.9)$ & $49.6(6.1)$ & $49.4(6.2)$ \\
\hline
\end{tabular}

Note: SD in parentheses.

Abbreviations: IMRT, intensity-modulated radiotherapy; MCS, mental component summary; PCS, Physical component summary; SF-8, 8-Item Short-Form Health Survey. 
Research and Reports in Urology is an international, peer-reviewed, open access journal publishing original research, reports, editorials, reviews and commentaries on all aspects of adult and pediatric urology in the clinic and laboratory including the following topics: Pathology, pathophysiology of urological disease; Investigation and treatment of urological disease; Pharmacology of drugs used for the treatment of urological disease. The manuscript management system is completely online and includes a very quick and fair peer-review system, which is all easy to use. Visit http://www.dovepress.com/testimonials.php to read real quotes from published authors

Submit your manuscript here: https://www.dovepress.com/research-and-reports-in-urology-journal 